\title{
Scope and limitations of a DMF bio-alternative within Sonogashira cross-coupling and Cacchi-type annulation
}

\author{
Kirsty L. Wilson ${ }^{1}$, Alan R. Kennedy ${ }^{1}$, Jane Murray ${ }^{2}$, Ben Greatrex ${ }^{3}$, Craig Jamieson ${ }^{1}$ \\ and Allan J. B. Watson*1
}

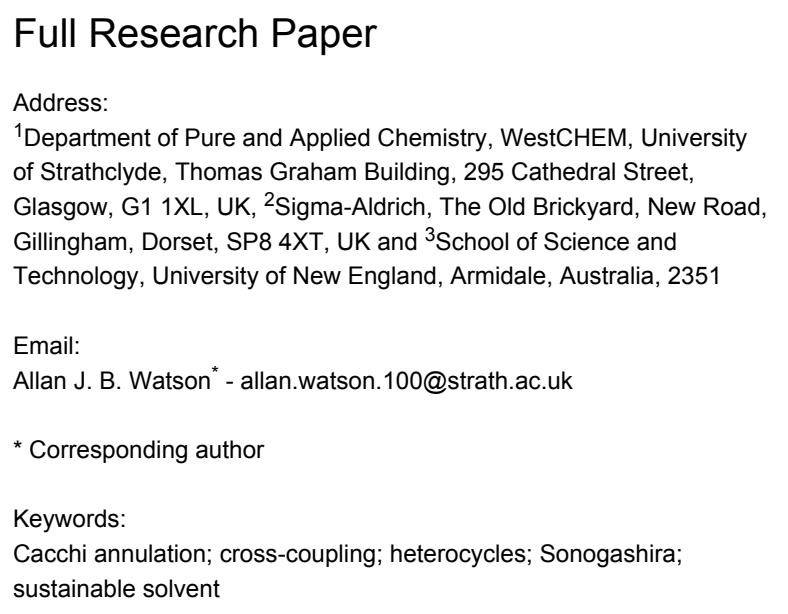

Beilstein J. Org. Chem. 2016, 12, 2005-2011. doi:10.3762/bjoc. 12.187

Received: 18 July 2016

Accepted: 22 August 2016

Published: 08 September 2016

This article is part of the Thematic Series "Green chemistry".

Guest Editor: L. Vaccaro

(c) 2016 Wilson et al.; licensee Beilstein-Institut. License and terms: see end of document.

\begin{abstract}
Pd-catalysed $\mathrm{C}-\mathrm{C}$ bond formation is an essential tool within the pharmaceutical and agrochemical industries. Many of these reactions rely heavily on polar aprotic solvents; however, despite their utility, these solvents are incompatible with the drive towards more sustainable chemical synthesis. Herein, we describe the scope and limitations of an alternative to DMF derived from renewable sources $\left(\right.$ Cyrene $\left.^{\mathrm{TM}}\right)$ in Sonogashira cross-coupling and Cacchi-type annulations.
\end{abstract}

\section{Introduction}

The Sonogashira reaction $[1,2]$ (Scheme 1) is a robust and broadly applicable Pd-catalysed bond-forming process that, alongside the Suzuki-Miyaura reaction [3], has steadily become an indispensible tool for $\mathrm{C}-\mathrm{C}$ bond formation in the pharmaceutical industry [4]. While the Sonogashira reaction can be effec-

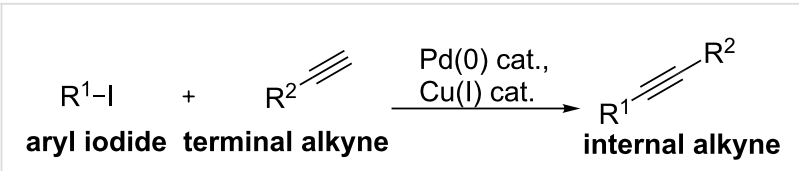

Scheme 1: The Sonogashira reaction. tively carried out in a variety of media [1,2], in the general sense this process clearly relies upon the use of dipolar aprotic solvents, in particular DMF. Indeed, some $41 \%$ of all Sonogashira reactions reported using aryl iodides can be linked to the use of DMF as a solvent [5].

In this context, the sustainability movement within pharmaceutical research and development strives to substitute solvents that have regulatory and environmental issues for those with a lower perceived risk. Indeed, solvent replacement has been designated a key research area with numerous pharmaceutical companies detailing their efforts towards a more sustain- 
able solvent selection as part of their overall sustainability programmes [6-23].

Based on its associated regulatory issues [24], it is perhaps no surprise that DMF continues to be a priority solvent for replacement. With legislation surrounding the use of DMF becoming increasingly stringent [24], numerous efforts have been made towards the use of alternative media in the Sonogashira reaction [25-30]. However, notwithstanding its issues, DMF is an excellent solvent for the Sonogashira reaction and its replacement frequently occurs at the expense of increased temperature (and therefore potentially substrate compatibility), reaction time, catalyst loading or the requirement for non-commercial/ expensive catalysts, and yield [25-30]. Consequently, poor choice of solvent replacement can result in one of industry's workhorse reactions becoming rather less predictable and robust.

In this regard, dihydrolevoglucosenone (Cyrene, Figure 1), accessed in two steps from cellulose [31,32], has been shown to possess similar physical properties to those of DMF and other dipolar aprotic solvents $[31,32]$. In addition to its renewability, Cyrene, as yet, has no associated pernicious effects and could potentially represent a direct and functional replacement in many of the fundamental reactions that typically employ DMF $[31,32]$. The replacement of solvents with regulatory issues with bio-derived alternatives has provided a series of advances within the cross-coupling arena [33], allowing efficient $\mathrm{C}-\mathrm{C}$ bond formation via cornerstone Pd-based methods including Suzuki-Miyaura [34,35], Mizoroki-Heck [36,37], Sonogashira [38], Stille [39], Hiyama reactions [40], and hydroformylation reactions [41].

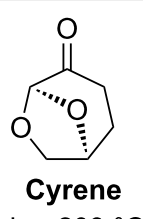

- bp: $203^{\circ} \mathrm{C}$

- Density: $1.25 \mathrm{~g} / \mathrm{mL}$

- Dipolarity $\left(\pi^{*}\right): 0.93$

Figure 1: Cyrene vs. DMF - selected physical properties [31,32].

In the current study, we present the use of Cyrene as an alternative solvent (direct DMF replacement) for the Sonogashira reaction, as well as related Cacchi-type annulations [42,43], with an emphasis on scope and limitations of its application.

\section{Results and Discussion}

To explore the use of Cyrene in the context of the Sonogashira cross-coupling, we established a simple benchmark reaction using iodobenzene (1a) and phenylacetylene (2a) (Table 1). A typical literature-derived catalyst system was employed $\left(\mathrm{Pd}\left(\mathrm{PPh}_{3}\right)_{2} \mathrm{Cl}_{2}\right.$ with $\mathrm{CuI}$ additive $\left.[44,45]\right)$ and conversion to diphenylacetylene (3a) was monitored.

Table 1: Reaction optimisation and comparison with existing solvents. ${ }^{\text {a }}$

a1 (1 equiv, $0.25 \mathrm{mmol}$ ), 2 (1.05 equiv, $0.26 \mathrm{mmol}$ ), $\mathrm{Pd}\left(\mathrm{PPh}_{3}\right)_{2} \mathrm{Cl}_{2}$ ( $2 \mathrm{~mol} \%)$, Cul (4 mol \%), base (see table), Cyrene, temperature (see table), time (see table), $\mathrm{N}_{2}$. ${ }^{\mathrm{b}}$ Isolated yield. ${ }^{\mathrm{C}}$ Reaction mixture solidified, product was not isolated. ${ }^{\mathrm{d}} \mathrm{THF}$ used as solvent. ${ }^{\mathrm{e} D M F}$ used as solvent.

Pleasingly, high conversion to product was immediately observed at room temperature in $5 \mathrm{~h}(94 \%$, Table 1 , entry 1$)$. This high conversion was consistent across several reaction concentrations (Table 1, entries 2 and 3 ) allowing for a reduction in solvent volume, commensurate with the principles of green chemistry $[46,47]$.

In attempts to further limit waste, we scanned a series of bases (see Supporting Information File 1); organic bases consistently performed more effectively and alternatives to $\mathrm{Et}_{3} \mathrm{~N}$ provided no significant advantages. However, during this process we identified some potential limitations of this emerging solvent. Specifically, inorganic bases such as $\mathrm{K}_{3} \mathrm{PO}_{4}$ and $\mathrm{Cs}_{2} \mathrm{CO}_{3}$ (Table 1, entries 4 and 5) resulted in the generation of a solid reaction mixture. Further analysis revealed that the aldol products $4 \mathbf{a}$ and $\mathbf{4 b}$ (Figure 2) were generated under specific reaction conditions.

The manufacturers note that when using Cyrene, materials to avoid are strong acids, and strong oxidising and reducing agents. Since sensitivity to base was not specified, we surveyed a range of bases at various temperatures to evaluate the limitations of Cyrene under such conditions (Table 2). 


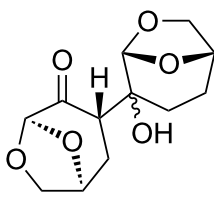

$4 a$

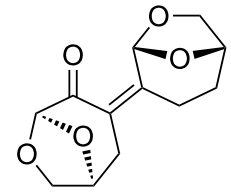

$4 b$

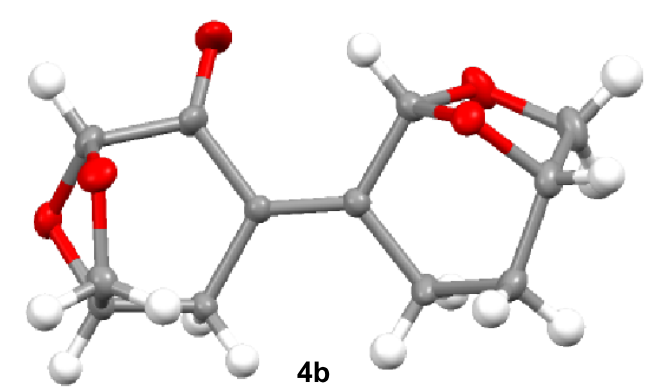

Figure 2: Aldol products $\mathbf{4 a}$ and $\mathbf{4 b}$ and single crystal X-ray structure of $\mathbf{4 b}$

Table 2: Evaluation of the base sensitivity of Cyrene. ${ }^{a}$

\begin{tabular}{|c|c|c|c|}
\hline Entry & Base & Temp. $\left({ }^{\circ} \mathrm{C}\right)$ & Reaction $(\mathrm{Y} / \mathrm{N})^{\mathrm{b}}$ \\
\hline & & 25 & $\mathrm{~N}$ \\
\hline \multirow[t]{3}{*}{1} & KOAC & 50 & $\mathrm{Y}$ \\
\hline & & 100 & $Y$ \\
\hline & & 25 & $\mathrm{~N}$ \\
\hline \multirow[t]{3}{*}{2} & Pyridine & 50 & $Y$ \\
\hline & & 100 & $Y$ \\
\hline & & 25 & $\mathrm{Y}$ \\
\hline \multirow[t]{3}{*}{3} & $\mathrm{~K}_{2} \mathrm{CO}_{3}$ & 50 & $\mathrm{Y}$ \\
\hline & & 100 & $\mathrm{Y}$ \\
\hline & & 25 & $\mathrm{~N}$ \\
\hline \multirow[t]{3}{*}{4} & DIPEA & 50 & $\mathrm{~N}$ \\
\hline & & 100 & $\mathrm{Y}$ \\
\hline & & 25 & $\mathrm{Y}$ \\
\hline \multirow[t]{3}{*}{5} & $\mathrm{Cs}_{2} \mathrm{CO}_{3}$ & 50 & $\mathrm{Y}$ \\
\hline & & 100 & $Y$ \\
\hline & & 25 & $\mathrm{~N}$ \\
\hline \multirow[t]{3}{*}{6} & $\mathrm{Et}_{3} \mathrm{~N}$ & 50 & $\mathrm{~N}$ \\
\hline & & 100 & $\mathrm{Y}$ \\
\hline & & 25 & $Y$ \\
\hline \multirow[t]{3}{*}{7} & $\mathrm{~K}_{3} \mathrm{PO}_{4}$ & 50 & $\mathrm{Y}$ \\
\hline & & 100 & $\mathrm{Y}$ \\
\hline & & 25 & $\mathrm{Y}$ \\
\hline \multirow[t]{3}{*}{8} & DBU & 50 & $Y$ \\
\hline & & 100 & $\mathrm{Y}$ \\
\hline & & 25 & $\mathrm{Y}$ \\
\hline \multirow[t]{3}{*}{9} & $\mathrm{KOH}$ & 50 & $\mathrm{Y}$ \\
\hline & & 100 & $\mathrm{Y}$ \\
\hline & & 25 & $\mathrm{Y}$ \\
\hline \multirow[t]{3}{*}{10} & $t$-BuOK & 50 & $Y$ \\
\hline & & 100 & $\mathrm{Y}$ \\
\hline & & 25 & $\mathrm{Y}$ \\
\hline \multirow[t]{2}{*}{11} & $\mathrm{NaH}$ & 50 & $Y$ \\
\hline & & 100 & $Y$ \\
\hline
\end{tabular}

aReaction conditions: base $(0.07 \mathrm{mmol})$ and Cyrene $(0.5 \mathrm{~mL})$ stirred at the indicated temeperature for $24 \mathrm{~h}$ before analysis by TLC and ${ }^{1} \mathrm{H}$ NMR . ${ }^{b} Y=$ reaction occurs, $\mathrm{N}=$ no reaction. See Supporting Information File 1.
Under these specific reaction conditions, with the exception of $\mathrm{Et}_{3} \mathrm{~N}$ and DIPEA, there was a clear base sensitivity displayed by Cyrene in the presence of all bases when the temperature was elevated above $25{ }^{\circ} \mathrm{C}$. Organic bases such as pyridine (Table 2, entry 2), DIPEA (Table 2, entry 4), and $\mathrm{Et}_{3} \mathrm{~N}$ (Table 2, entry 6) were tolerated at $25{ }^{\circ} \mathrm{C}$ with DIPEA and $\mathrm{Et}_{3} \mathrm{~N}$ also tolerated at $50{ }^{\circ} \mathrm{C}$. DBU, however, was not tolerated at any temperature (Table 2, entry 8). With the exception of KOAc (Table 2, entry 1), all inorganic bases resulted in reaction with the solvent at room temperature (Table 2, entries 3, 5, 7, and 9-11). The extent of the reaction varied from the generation of additional components, such as $\mathbf{4 a}$ and $\mathbf{4 b}$, to gelation or complete solidification of the reaction mixture. However, in a moderately basic reaction mixture (e.g., using $\mathrm{Et}_{3} \mathrm{~N}$ ) at mild reaction temperatures this issue could be entirely avoided. As such, optimisation of the Sonogashira process allowed complete conversion and $96 \%$ isolated yield in $1 \mathrm{~h}$ at $30{ }^{\circ} \mathrm{C}$ (Table 1, entry 8). Importantly, the Cyrene-based system compared very favourably upon comparison with standard solvents (THF and DMF; Table 1, entries 9 and 10, respectively).

Continuing with the primary investigation and with an optimised set of reaction conditions, we sought to explore the generality of Cyrene in the Sonogashira cross-coupling (Scheme 2). Significantly, a broad range of functionalised aryl and heteroaryl iodides were tolerated (Scheme 2a).

In addition, electron-deficient aryl bromides were accommodated, although with some variation in yield (3c, 3i, 3o, 3n). Functionality on the alkyne component was also typically well tolerated (Scheme $2 b$ ). While $\mathbf{3 i}$ and $\mathbf{3 j}$ required an extended reaction time, this was a substrate-specific problem for the use of 2a with these ortho-substituted aryl iodides that was not apparent for other alkyne/ortho-substituted iodoarene combinations (Scheme 2c).

Judicious selection of reacting components also enabled the development of a useful Cacchi-type annulation (Scheme 3) 


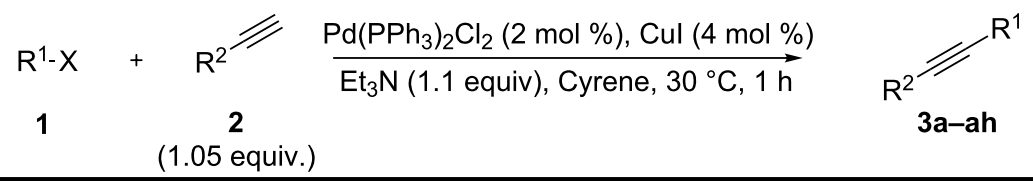<smiles>Fc1ccc(C#CPc2ccccc2)cc1</smiles>

3b: quant. $(X=I)$ $(96 \%)^{a}$<smiles>O=[N+]([O-])c1cccc(C#Cc2ccccc2)c1</smiles>

3h: $99 \%(X=1)$<smiles>O=[N+]([O-])c1ccc(C#Cc2ccccc2)cc1</smiles>

3c: quant. $(X=1)$ $28 \%(X=B r)$<smiles>COc1ccc(C#Cc2ccccc2)cc1</smiles>

3d: quant. $(X=I)$<smiles>Oc1ccc(C#Cc2ccccc2)cc1</smiles>

3e: $68 \%(X=I)^{b}$<smiles>COc1cccc(C#Cc2ccccc2)c1</smiles>

3f: $99 \%(X=1)$<smiles>Clc1cccc(C#Cc2ccccc2)c1</smiles>

3g: $82 \%(X=I)$<smiles>C(#Cc1ccc2cccnc2n1)c1ccccc1</smiles><smiles>Cc1ccccc1C#Cc1ccccc1</smiles><smiles>Clc1ccccc1C#Cc1ccccc1</smiles><smiles>C(#Cc1cccs1)c1ccccc1</smiles>
3i: $22 \%(X=I) \quad$ 3j: quant. $(X=I)^{\mathrm{C}}$ 3k: $92 \%(X=I)$<smiles>O=[N+]([O-])c1ccc(C#Cc2ccccc2)cn1</smiles><smiles>Clc1ccc(C#Cc2ccccc2)nc1</smiles>

31: $92 \%(X=B r)$ $83 \%(X=1)^{c}$<smiles>Clc1cccc(C#Cc2ccccc2)n1</smiles><smiles>Cn1ccc2cc(C#Cc3ccccc3)ccc21</smiles>

3n: $94 \%(X=B r)$

3o: quant. $(\mathrm{X}=\mathrm{Br})$

3p: $67 \%(X=1)$

b) Variation of the alkyne using aryl iodide $1 \mathrm{a}$<smiles>CC(C)(C)C#C[As]</smiles><smiles>CS(C)(C)C#Cc1ccccc1</smiles><smiles>[Se-]OCCC#Cc1ccccc1</smiles>
3s: $81 \%$<smiles>CN(C)CC#Cc1ccccc1</smiles>
3t: $60 \%$<smiles>CCCC#Cc1ccccc1</smiles>

3u: $96 \%$

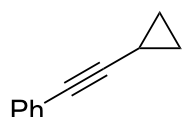

3v: $85 \%$ $(95 \%)^{\mathrm{a}}$<smiles>C(#Cc1ccccc1)c1ccccc1</smiles>

3w: $81 \%$<smiles>C(#Cc1ccccc1)C1=CCCCC1</smiles>

3x: quant.<smiles>Cc1ccc(C#CCOCCc2ccccc2)cc1</smiles><smiles>FC(F)(F)c1ccccc1C#Cc1ccccc1</smiles>

3z: $86 \%$<smiles>C(#Cc1ccccn1)c1ccccc1</smiles>

$\mathrm{Ph}$

3aa: $97 \%$<smiles>C(#Cc1cccs1)c1ccccc1</smiles>

3ab: $92 \%$

C) Heteroatom-substituted alkynes with ortho-substituted aryl iodides<smiles>CC(=O)c1ccccc1C#CC(C)(C)C</smiles>

3ac: $89 \%$<smiles>Cc1ccccc1C#C[Mg]C(C)(C)C</smiles>

3ad: 93\% $(92 \%)^{a}$<smiles>CCCCCCCCC(C)(C)C</smiles>

3ae: $85 \%$

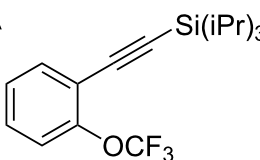

3af: $68 \%$<smiles>CC(C)[SiH2]C#Cc1ccccc1N</smiles>

3ag: $66 \%$<smiles>Clc1ccccc1C#C[AsH2]</smiles>

3ah: $65 \%$ reaction time.

$[42,43]$. Specifically, employing ortho-amino (5) or orthohydroxyaryl iodides (6) in the Sonogashira process generated an alkyne intermediate that, upon increasing the reaction temperature from $30{ }^{\circ} \mathrm{C}$ to $60{ }^{\circ} \mathrm{C}$, could undergo 5-endo-dig cyclisation to forge functionalised and pharmaceutically relevant indole, benzofuran, and aza-indole scaffolds in a single operation $(7 \mathbf{a}-\mathbf{f})[48-52]$.
Finally, with the viewpoint of generality of DMF substitution by Cyrene, the base/temperature sensitivity issue may have potential implications for further applications of Cyrene within well-used organic transformations. For example, the majority of many other standard cross-coupling processes employ inorganic or organic bases and heat (e.g., Suzuki-Miyaura, Heck). Accordingly, Cyrene may be projected to be incompatible with 


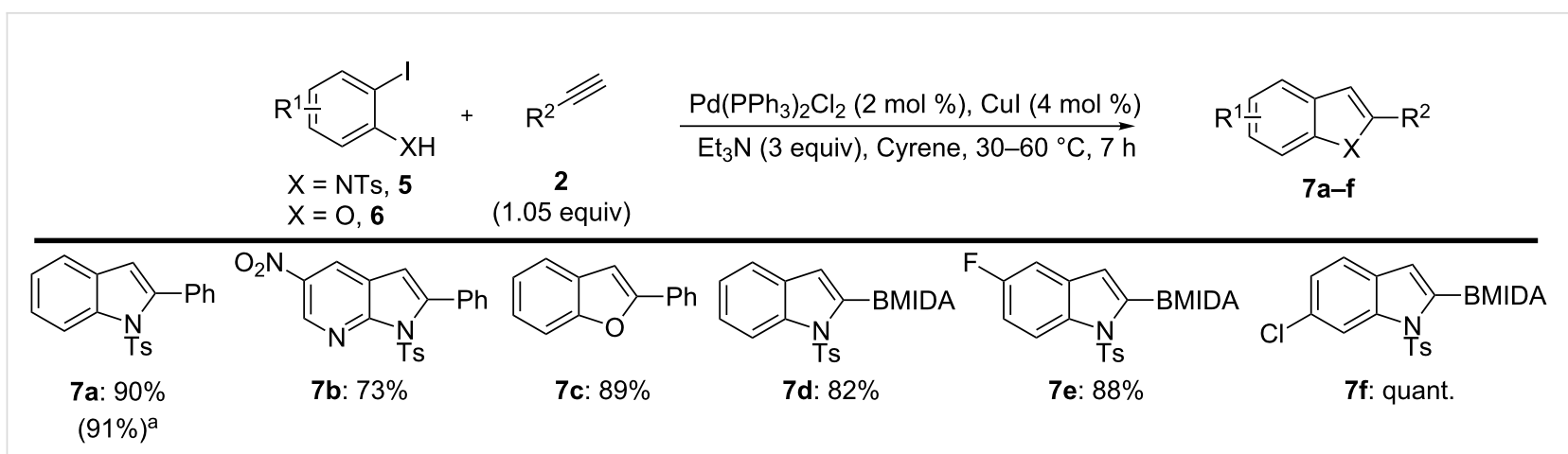

Scheme 3: Cacchi-type annulation of o-amino/hydroxy iodoarenes. Isolated yields. ${ }^{a}$ Yield using DMF as solvent.

standard conditions for these reactions and its use would necessitate base-free or exceptionally mildly basic reaction conditions. In contrast, amide-bond formation is the most practiced reaction in the pharmaceutical industry [4] and these are routinely performed in DMF at room temperature in the presence of organic bases [53]. As such, Cyrene may offer considerable potential in this area. However, additional work will be required to validate the practicality of Cyrene as a viable DMF replacement in these applications.

\section{Conclusion}

In summary, we have developed a mild and robust method for the Sonogashira reaction, employing the bio-derived and sustainable alternative to DMF, Cyrene. In addition, we have shown the capacity for extension of the utility of this new solvent towards enabling the cascade synthesis of functionalised indoles and benzofurans via a Cacchi-type annulation. Perhaps more importantly, we have documented some of the limitations of the use of Cyrene as a solvent, providing guidance emerging in relation to the thermal and chemical (base) stabilities of this promising green solvent.

\section{Supporting Information}

\section{Supporting Information File 1}

Experimental procedures, analytical data, copies of NMR spectra, and single X-ray crystal diffraction data of $\mathbf{4 b}$. [http://www.beilstein-journals.org/bjoc/content/ supplementary/1860-5397-12-187-S1.pdf]

\section{Acknowledgements}

"Sigma-Aldrich Company Limited" is a subsidiary of Merck KGaA. We thank the University of Strathclyde for a $\mathrm{PhD}$ studentship (KLW), Sigma-Aldrich for financial and material support, Circa for Cyrene, and the EPSRC UK National Mass Spectrometry Facility at Swansea University for analyses.

\section{References}

1. Chinchilla, R.; Nájera, C. Chem. Soc. Rev. 2011, 40, 5084-5121. doi:10.1039/c1cs15071e

2. Chinchilla, R.; Nájera, C. Chem. Rev. 2007, 107, 874-922. doi:10.1021/cr050992x

3. Miyaura, N.; Suzuki, A. Chem. Rev. 1995, 95, 2457-2483. doi:10.1021/cr00039a007

4. Roughley, S. D.; Jordan, A. M. J. Med. Chem. 2011, 54, 3451-3479. doi:10.1021/jm200187y

5. A SciFinder search of Sonogashira reaction of aryl iodides revealed that of 341979 reactions, 140997 were performed in DMF. Search conducted 7th June 2016.

6. Breeden, S. W.; Clark, J. H.; Macquarrie, D. J.; Sherwood, J. Green solvents. In Green Techniques for Organic Synthesis and Medicinal Chemistry; Zhang, W.; Cue, B. W., Jr., Eds.; Wiley: Chichester, United Kingdom, 2012.

7. Prat, D.; Hayler, J.; Wells, A. Green Chem. 2014, 16, 4546-4551. doi:10.1039/C4GC01149J

8. Eastman, H. E.; Jamieson, C.; Watson, A. J. B. Aldrichimica Acta 2015, $48,51-55$.

9. Curzons, A. D.; Constable, D. C.; Cunningham, V. L. Clean Prod. Process. 1999, 1, 82-90.

10. Jiménez-González, C.; Curzons, A. D.; Constable, D. J. C.; Cunningham, V. L. Clean Technol. Environ. Policy 2004, 7, 42-50. doi:10.1007/s10098-004-0245-z

11. Constable, D. J. C.; Dunn, P. J.; Hayler, J. D.; Humphrey, G. R.; Leazer, J. L., Jr.; Linderman, R. J.; Lorenz, K.; Manley, J.; Pearlman, B. A.; Wells, A.; Zaks, A.; Zhang, T. Y. Green Chem. 2007, 9, 411-420. doi:10.1039/B703488C

12. Alfonsi, K.; Colberg, J.; Dunn, P. J.; Fevig, T.; Jennings, S.; Johnson, T. A.; Kleine, H. P.; Knight, C.; Nagy, M. A.; Perry, D. A.; Stefaniak, M. Green Chem. 2008, 10, 31-36. doi:10.1039/B711717E

13. http://www.acs.org/content/dam/acsorg/greenchemistry/industriainnova tion/roundtable/solvent-selection-guide.pdf. AstraZeneca's guidance.

14. Henderson, R. K.; Jiménez-González, C.; Constable, D. J. C.; Alston, S. R.; Inglis, G. G. A.; Fisher, G.; Sherwood, J.; Binks, S. P.; Curzons, A. D. Green Chem. 2011, 13, 854-862. doi:10.1039/c0gc00918k

15. Prat, D.; Pardigon, O.; Flemming, H.-W.; Letestu, S.; Ducandas, V.; Isnard, P.; Guntrum, E.; Senac, T.; Ruisseau, S.; Cruciani, P.; Hosek, P. Org. Process Res. Dev. 2013, 17, 1517-1525. doi:10.1021/op4002565 
16. Byrne, F. P.; Jin, S.; Paggiola, G.; Petchey, T. H. M.; Clark, J. H.; Farmer, T. J.; Hunt, A. J.; McElroy, C. R.; Sherwood, J. Sustainable Chem. Processes 2016, 4, 7. doi:10.1186/s40508-016-0051-z

17. Alder, C. M.; Hayler, J. D.; Henderson, R. K.; Redman, A. M.; Shukla, L.; Shuster, L. E.; Sneddon, H. F. Green Chem. 2016, 18, 3879-3890. doi:10.1039/C6GC00611F

18. Chandrasekhar, S.; Narsihmulu, C.; Shameem Sultana, S.; Ramakrishna Reddy, N. Org. Lett. 2002, 4, 4399-4401. doi:10.1021/ol0266976

19. MacMillan, D. S.; Murray, J.; Sneddon, H. F.; Jamieson, C.; Watson, A. J. B. Green Chem. 2012, 14, 3016-3019. doi:10.1039/c2gc36378j

20. Taygerly, J. P.; Miller, L. M.; Yee, A.; Peterson, E. A. Green Chem. 2012, 14, 3020-3025. doi:10.1039/c2gc36064k

21. MacMillan, D. S.; Murray, J.; Sneddon, H. F.; Jamieson, C.; Watson, A. J. B. Green Chem. 2013, 15, 596-600. doi:10.1039/c2gc36900a

22. McGonagle, F. I.; MacMillan, D. S.; Murray, J.; Sneddon, H. F.; Jamieson, C.; Watson, A. J. B. Green Chem. 2013, 15, 1159-1165. doi:10.1039/c3gc40359a

23. Skowerski, K.; Białecki, J.; Tracz, A.; Olszewski, T. K. Green Chem. 2014, 16, 1125-1130. doi:10.1039/C3GC41943F

24. European Chemicals Agency (ECHA), Candidate List of Substances of Very High Concern for Authorisation. http://echa.europa.eu/candidate-list-table (accessed May 23, 2016).

25. Fleckenstein, C. A.; Plenio, H. Green Chem. 2008, 10, 563-570. doi:10.1039/b800154e

26. Bakherad, M. Appl. Organomet. Chem. 2013, 27, 125-140. doi:10.1002/aoc.2931

27. Ibrahim, M. B.; Ali, B. E.; Malik, I.; Fettouhi, M. Tetrahedron Lett. 2016, 57, 554-558. doi:10.1016/j.tetlet.2015.12.086

28. Gonçalves, R. S. B.; de Oliveira, A. B. V.; Sindra, H. C.; Archanjo, B. S.; Mendoza, M. E.; Carneiro, L. S. A.; Buarque, C. D.; Esteves, P. M. ChemCatChem 2016, 8, 743-750. doi:10.1002/cctc.201500926

29. Camp, J. E.; Dunsford, J. J.; Dacosta, O. S. G.; Blundell, R. K.; Adams, J.; Britton, J.; Smith, R. J.; Bousfield, T. W.; Fay, M. W. RSC Adv. 2016, 6, 16115-16121. doi:10.1039/C5RA25712C

30. McAfee, S. M.; Cann, J. R.; Josse, P.; Blanchard, P.; Cabanetos, C.; Welch, G. C. ACS Sustainable Chem. Eng. 2016, 4, 3504-3517. doi:10.1021/acssuschemeng.6b00554

31. Sherwood, J.; De Bruyn, M.; Constantinou, A.; Moity, L.; McElroy, C. R.; Farmer, T. J.; Duncan, T.; Raverty, W.; Hunt, A. J.; Clark, J. H. Chem. Commun. 2014, 50, 9650-9652. doi:10.1039/C4CC04133J

32. Koseki, K.; Ebata, T.; Kawakami, H.; Matsushita, H.; Itoh, K.; Naoi, Y. Method of producing (S)-4-hydroxymethyl-y-lactone. U.S. Patent 5112994, May 12, 1992.

33. Ilgen, F.; König, B. Green Chem. 2009, 11, 848-854. doi:10.1039/b816551c

34. Azua, A.; Mata, J. A.; Heymes, P.; Peris, E.; Lamaty, F.; Martinez, J.; Colacino, E. Adv. Synth. Catal. 2013, 355, 1107-1116. doi:10.1002/adsc.201201047

35. Wan, J.-P.; Wang, C.; Zhou, R.; Liu, Y. RSC Adv. 2012, 2, 8789-8792. doi:10.1039/c2ra21632a

36. Strappaveccia, G.; Ismalaj, E.; Petrucci, C.; Lanari, D.; Marrocchi, A.; Drees, M.; Facchetti, A.; Vaccaro, L. Green Chem. 2015, 17, 365-372. doi:10.1039/C4GC01677G
37. Rasina, D.; Kahler-Quesada, A.; Ziarelli, S.; Warratz, S.; Cao, H.; Santoro, S.; Ackermann, L.; Vaccaro, L. Green Chem., in press. doi:10.1039/C6GC01393G

38. Strappaveccia, G.; Luciani, L.; Bartollini, E.; Marrocchi, A.; Pizzo, F.; Vaccaro, L. Green Chem. 2015, 17, 1071-1076. doi:10.1039/C4GC01728E

39. Imperato, G.; Vasold, R.; König, B. Adv. Synth. Catal. 2006, 348, 2243-2247. doi:10.1002/adsc.200600248

40. Ismalaj, E.; Strappaveccia, G.; Ballerini, E.; Elisei, F.; Piermatti, O.; Gelman, D.; Vaccaro, L. ACS Sustainable Chem. Eng. 2014, 2, 2461-2464. doi:10.1021/sc5004727

41. Pongrácz, P.; Kollár, L.; Mika, L. T. Green Chem. 2016, 18, 842-847. doi:10.1039/C5GC01778E

42. Cacchi, S.; Fabrizi, G. Chem. Rev. 2011, 111, PR215-PR283. doi:10.1021/cr100403z

43. Humphrey, G. R.; Kuethe, J. T. Chem. Rev. 2006, 106, 2875-2911. doi: $10.1021 / \mathrm{cr} 0505270$

44. Stuart, D. R.; Bertrand-Laperle, M.; Burgess, K. M. N.; Fagnou, K. J. Am. Chem. Soc. 2008, 130, 16474-16475. doi:10.1021/ja806955s

45. Chen, X.; Wu, Y.; Xu, J.; Yao, H.; Lin, A.; Huang, Y. Org. Biomol. Chem. 2015, 13, 9186-9189. doi:10.1039/C5OB01338K

46. Anastas, P. T.; Warner, J. C. Green Chemistry: Theory and Practice; Oxford University Press: New York, NY, U.S.A., 1998; p 30.

47. Anastas, P.; Eghbali, N. Chem. Soc. Rev. 2010, 39, 301-312. doi:10.1039/B918763B

48. Heinrich, T.; Seenisamy, J.; Emmanuvel, L.; Kulkarni, S. S.; Bomke, J.; Rohdich, F.; Greiner, H.; Esdar, C.; Krier, M.; Grädler, U.; Musil, D. J. Med. Chem. 2013, 56, 1160-1170. doi:10.1021/jm3016014

49. Hong, S.; Kim, J.; Seo, J. H.; Jung, K. H.; Hong, S.-S.; Hong, S. J. Med. Chem. 2012, 55, 5337-5349. doi:10.1021/jm3002982

50. Hong, S.; Lee, S.; Kim, B.; Lee, H.; Hong, S.-S.; Hong, S. Bioorg. Med. Chem. Lett. 2010, 20, 7212-7215. doi:10.1016/j.bmcl.2010.10.108

51. Tang, J.; Hamajima, T.; Nakano, M.; Sato, H.; Dickerson, S. H.; Lackey, K. E. Bioorg. Med. Chem. Lett. 2008, 18, 4610-4614. doi:10.1016/j.bmcl.2008.07.019

52. Seath, C. P.; Wilson, K. L.; Campbell, A.; Mowat, J. M.; Watson, A. J. B. Chem. Commun. 2016, 52, 8703-8706. doi:10.1039/C6CC04554E

53. El-Faham, A.; Albericio, F. Chem. Rev. 2011, 111, 6557-6602. doi:10.1021/cr100048w 


\section{License and Terms}

This is an Open Access article under the terms of the Creative Commons Attribution License

(http://creativecommons.org/licenses/by/4.0), which permits unrestricted use, distribution, and reproduction in any medium, provided the original work is properly cited.

The license is subject to the Beilstein Journal of Organic Chemistry terms and conditions:

(http://www.beilstein-journals.org/bjoc)

The definitive version of this article is the electronic one which can be found at:

doi:10.3762/bjoc.12.187 